\title{
Regulatory System: Safety and Efficacy of Vaccines
}

Merita Kucuku*

National Agency for Medicines and Medical Devices, Tirana, Albania

*Corresponding author: Merita Kucuku, National Agency for Medicines \& Medical Devices, Tirana, Albania, E-mail:emkoni@hotmail.com

Received date: December 08, 2017; Accepted date: December 19, 2017; Published date: December 26, 2017

Copyright: (C2017 Kucuku M. This is an open-access article distributed under the terms of the Creative Commons Attribution License, which permits unrestricted use, distribution, and reproduction in any medium, provided the original author and source are credited.

\begin{abstract}
Today the types and number of vaccines in immunization schedule and in the market are increasing. The vaccination applied at first day of life and continues in different ages during life, in healthy people and by many states is mandatory for school entry requirements. It is the reason that the safety and efficacy of vaccines are very important and the much discussed issue in the world. Some country time after time face unfavorable situation for vaccination. The technology of information is very developed and people can get information for immunization and vaccines in different ways as journal, internet, TV, social media, newspaper, discussion between each other etc. which have an impact. Not always the information is right, but unfortunately this can cause panic and damage the system of immunization in the country and sometimes in the world. It is very important to prepare the situation in advance which mean not to allow space for misunderstanding and misinterpretation of vaccines safety and efficacy. World Health Organization (WHO) coordinates efforts directed towards achieving international consensus, but the National Regulatory Authority (NRAs) ultimately makes decisions based on benefit/ risk assessment for their population. Strengthening and increasing the effectiveness of the NRA function is an immediate task.
\end{abstract}

Keywords Vaccine; Regulatory system; Safety; Efficacy

\section{Introduction}

The regulatory system has a responsibility to protect and promote the public health, to ensure the safety and efficacy of vaccines because the injection of ineffective and poor quality of products sometimes can cause very serious problems even and death and undermine confidence in the health system, health professionals, pharmaceutical manufacturers and distributors. Another problem of poor quality and non-effective vaccines has a financial wasted cost. National Regulatory Authority (NRA) or National Control Laboratories (NLC) are different and their consideration for benefit/ risk in their country is different based on vaccine supply, disease prevalence and severity, their specific epidemiological situation, which must be taken in consideration when making a decision [1].

After marketing the vaccines is necessary to have attention for safety and efficacy and all data reported need to treat properly. NRA during this time must have all information about vaccines, production, producer, doses injected, package, all information for patients reported and the area where is injected. The passive surveillance can help for safety of signals of adverse events following immunization (AEFI).

\section{What about the regulation system?}

The regulation system incorporates several mutually reinforcing activities all aimed at promoting and protecting public health which vary from country to country in scope and implementation which include the function:

- Licensing, in case of imported vaccines in non-production countries all vaccines are imported from other countries and licensing is based on protocol of summary product

- Assessing the safety, efficacy and quality of vaccines and issuing marketing authorization.
- Inspecting and surveillance of importers, wholesalers of vaccines

- Controlling and monitoring the quality of vaccines on the market. If any suspicion is for quality of vaccines in the market can perform the quality control in the control lab

- Monitoring safety of marketed vaccines, including collecting and analyzing adverse reaction reports

-Providing independent information on vaccines to professionals and public. The information must be very clearly because have important impact.

Regulatory functions involve interactions with various stakeholders (e.g. manufacturers and traders, and consumers, and health professionals, and researchers and governments) whose economic, social and political motives may differ, making implementation of regulation both politically and technically challenging [2].

\section{Material and Methods}

This article is based on various experiences of regulatory authorities regarding the safety and efficacy of vaccines.

Regulatory oversight is critical to allow access to vaccines that are safe, effective and of assuring quality [3].

The vaccines are biological products that injected in healthy people. Before injection the vaccines must meet three criteria for Good Quality and Safe, and Effective which will judge based on strong scientific arguments. It is necessary the NRA in the country to be effective and this depends from different factors. General factors for criteria include political will and commitment to regulation, strong public support for Adverse Event Following Immunization (AEFI) reporting, effective cooperation between NRA and other government institutions, including customs, police and sufficient qualified staff involved in the judgment of the situation. 
The NRA should have a clear mission, clearly defined roles and responsibilities, adequate and sustainable financial resources, international collaboration between NRAs which also is important. All countries are using vaccines for the vaccination of their population and the safety of vaccines is a public health issue. While the people vaccinate, sometimes AEFI happen. In this situation question is often asked: are these experiences related to vaccines? It is important to answer this question because the patients and healthcare workers should be cleared what is happening because very often the issue is not related to the vaccine. Some parents understand the benefits of immunization and some have concern about the safety for example public concern about the safety of whole -cell pertussis vaccine in the 1980 s, which resulted in decreased vaccine coverage and the return of epidemic disease in Japan, Sweden, United Kingdom and other countries. In 2008 more measles cases were reported than in any year since 1997 and from them more than 90\% of those infected had not been or their vaccination status was unknown. California during January 1 to June 30 year 2010, were reported 1337 pertussis cases to the California Department of Public Health a $41,8 \%$ increase from 258 cases reported during the same period in 2009 [4]. The safety profile of vaccines includes: clinical trial, post marketing surveillance. The information on the safety from clinical trials is limited because: The small numbers of subjects in clinical trials to identify the rare adverse events that occur in ratiol: 10000 or less. Case series analysis, various methods of signal detection, registries and observational (nonrandomized), post approval scientific and data gathering activities following the use of vaccines in the population. During the clinical trial the actions are correctly no any mistake during immunization, but in real life we are not sure if the healthcare is acting correctly. Restricted population in terms of age, gender and ethnicity, restricted comorbidity, restricted co-medication, restricted conditions of use.

Relatively short duration of exposure and follow up and the statistical problems associated with looking at multiply outcomes. Always the vaccines are authorized when the ratio benefit/risk is judged positive for the target population, but not every time the risk is identified on time, maybe the risk is greater than that the target group.

The efficacy of vaccines varies in different countries and according to Maria Rosario Capeding et al efficacy by country was consistent with the overall estimate ranging from $51.1 \%$ in Vietnam to $79.0 \%$ in Malaysia [5]. At 9 centers in Columbia, 5 centers in Brazil 5 centers in Mexico, 2 centers in Puerto Rico and 1 center in Honduras from June 2011 through March 2012 enrolled healthy children between ages of 9 and 16 years, where received the vaccine 13920 and 6949 received placebo. A total of 2000 of these children were assigned to the reactogenicity and immunogenicity subgroup: 1334 in the vaccine group and 666 in the control group were similar with respect to age and sex ratio. More than $95 \%$ of participants in each group received all three injections and $90 \%$ in each group were included in the per protocol efficacy analysis. The participant in each group: Colombia 9743 and 921 in the subgroup, Mexico 3464 and 32 in subgroup, Mexico 3464 and 327 in subgroup, Honduras 2799 and 300 in the subgroup and Puerto Rico 1315 and 152 in subgroup. More than 95\% of participants in each group received all three injections and $90 \%$ were included in the per protocol efficacy analysis. In the control group the overall incidence of virologically confirmed Dengue (VCD) was 3,8 cases per 100 person- years at risk between months 13 and 25 and 2,9 cases per 100 persons; $n$ - years during the entire 25 month period. The vaccine efficacy was $60.8 \%$ (95\% confidence interval, CI) 52,0 to $68.0 \%$ on the basis of 176 cases of VCD in the vaccine group and 221 in the control group that were diagnosed more than 28 days after the third dose. Efficacy was highest for serotype 4 and lowest for serotype 2 and similar in two age groups and was $83,7 \%$ (95\% CI, 62,2 to 93,7) among children who had antibodies against dengue at baseline and also varied according countries. 17 hospitalizations for VCD per 6 days after at least 1 injection in vaccine group was compared with 43 hospitalizations per 4 days in the control group for a vaccine efficacy of $80,3 \%$ (95\% CI, 64,7 to 89,5$) .12$ cases in of severe dengue: 1 in the vaccine group (serotype 1) and 11 in the control group ( 3 cases of serotype1, 4 cases of serotype 2, 3 cases of serotype 3 and 1 cases of serotype 4. Efficacy against severe dengue after first injection was $95,5 \%(95 \%$ CI, 66,8 to 99,9$)$ and $90 \%$ (95\% CI, 10,7 to 99,8$)$ after the third injection and single episode based only on lab results was classified as severe but the child was not hospitalized [6].

Almost in all countries are established the Pharmacovigilenca system which includes in regulatory agencies or in some countries are and special agency which is involved in AEFI cases.

\section{Why need post marketing surveillance}

As Francois Verdier mentioned Rotashield vaccine the perfect example for explanation of the risk/benefit ratio of vaccination, which in general is very different between developing countries and developed countries. The product has been withdrawn in the US after cases of intussusceptions in temporal relation to the administration of the vaccine [7].

We can mention also these cases happened in different countries in different times to explain the importance of post marketing surveillance.

Kyoto incident related to diphtheria vaccine on November 1948 where 15561 children from infants to 13 years old received the second injection of diphtheria vaccine (precipitated the diphtheria toxoid). The first injection of the vaccine, given 2 weeks ago, had no problem and 1-2 days after the second injection, 606 children showed severe adverse reactions as edema or ulcer at the injection site. Among 150 children hospitalized and 68 died. Nine of died showed acute paralysis characteristic to diphtheria, $80 \%-90 \%$ of the dead were infants under 2 years old. This accident happened with contaminated vaccine with incompletely inactivated toxin and contamination was from the poor homogenization of the final bulk [8].

Lubeck incident related to the accident in BCG vaccine, where 251 neonates were orally given three doses of the new Bacille CalmetteGuerin (BCG) anti tuberculosis vaccines contaminated with Mycobacterium tuberculosis. A total of 173 infants developed clinical or radiological signs of $\mathrm{TB}$, but survived the infection, while 72 died from TB. Some blamed the accident on BCG itself by postulating reversion to full virulent, such a possibility was conclusively disproven, by combining microbiological, clinical, and epidemiological data the chief public health investigator Moegling concluded that the BCG vaccine had been contaminated variable amounts of fully virulent $M$. turbeculosis [9].

With more than 200000 children in five western and mid- western USA states received a polio vaccine in which the process of inactivating the live virus proved to be defective with 40000 cases of polio and 200 children with varying degrees of paralysis and killing 10 [10].

SV4O in polio vaccine evidenced are from the mid 1900 and 1960 and 1970 and it is important to know for regulators to be prepared if they were to occur in the future for reaching a globally convergent approach to regulatory evaluation. Each time new data emerge a new 
Page 3 of 3

benefit/risk assessment might be necessary. SV40 can infect and the nasal, and oral mucosal routes live virus could be recovered 11 days after inoculation. In May 1961 the NIH convened the ICPV to discuss the SV40 issue which had been found in both IPV (licensed product) and OPV (investigational product), but was no evidence that small amount of SV40 are capable of producing diseases in humans. In 1984 the Institute of Medical Biology developed a test to screen Rhesus Monkeys for SV40, which found to be positive was not permitted to be used for vaccine production.

Bacteriophage in live viral vaccines as a contaminant of bovine sera in MMR lives in 1972. Only one phage $\varphi$-1 was isolated from vaccines.

Reverse Transcriptase (RT) in measles and other vaccines in 1995. There was a lack of evidence for any real health concern remained such as the possibility of the endogenous retroviral particles chicken cells and it was recommended. Also, no evidence to indicate that RT activity found using the Product Enhanced Reverse Transcriptase assay had any medical significance for humans or that reflected the presence of transmissible retrovirus in the vaccines.

Porcine circovirus (PCV) and porcine circovirus DNA sequences in rotavirus vaccine. According to EMA CHMP considered that PCV findings did not present a threat to public health and consequently that there was no need to restrict the use of the vaccines and concluded that the benefit 0 risk balance of both vaccines and potential human circovirus with porcine circoviruses as human circoviruses are unknown. On 2011 are vaccinated 112 million doses rotarix distributed worldwide and no safety signal has been identified in humans vaccinated with rotarix that could be assessed as being related to a potential PCV -1 infection.

- Not sufficient inactivation of polio virus (Cutter Co USA)

What is important to know if something is happening with the quality of vaccine?

The important thing to know if something happened with the quality of vaccines is initial findings that suggested the presence of an adventitious agent, background information on the agent or signal, follow-up steps that were taken by relevant organizations, scientific advice that was sought, regulatory issues and actions that were taken, vaccine supply implications, public transparency and communication with other organizations, public health and other issues, the overall outcome of the event, lesson learned. The important thing in every country is to have the Plan of Management Risk. The management of single risk consists of 4 steps as risk assessment, risk minimization, risk communication. A vaccine has multiple risks attached to it and individual risk with varying in terms of severity and individual patients and public health impact. The concept of risk management must also consider the combination of information on multiple risks with the aim of ensuring that the benefits exceed the risks by the greatest possible margin individual patients and population level [11].

Activities of pharmacovigilance are defined to identify, characterize, prevent or minimize risk related to vaccines and to assess their effectiveness.

\section{Results and Discussion}

The spontaneous reporting increases the sensitivity for risk identification or confirms, characterise or quantify possible hazards.
Post- marketing data bases is very valuable sources of safety information for assessing of potential risk, while active surveillance has a financial cost and not every country can do it.

The data and reports should collect, in agreed intervals and in appropriate format of $\mathrm{PV}$

Epidemiologic method is a key component in the evaluation of adverse events, case- control studies (retrospective and prospective). The decision making of the assessment is the benefit/ risk and based on the data of this ration can change the assessment. The Different countries may have different benefit/risk consideration for their country which is based on vaccine supply, disease prevalence, severity, specific epidemiological situation and these factors must be taken in consideration for decision making.

\section{Conclusions}

Strengthening of regulatory function in every country and collaboration of all healthcare staff in country avoids misinterpretation and misunderstanding of safety and efficacy of vaccines. Exchange experiences and sharing information between regulatory agencies for issues related to the safety and efficacy of vaccines can prepare the situation in advance and protect immunization system and public confidence and remove obstacles. It is necessary a good communication between all stakeholders for safety and efficacy of vaccines. We should not neglect the experiences of the past, but to serve as a lesson to be cautious about the inconsistency in the future for stronger immunization system.

\section{References}

1. Van Boxtel CJ, Santoso B, Edwards R (2002) Drug Benefits and risks, revised 2nd edition 2008. Br J Clin Pharmacol 54: 186.

2. Kucuku M (2012) Role of Pharmacovigilance on Vaccines Control. J Rural Med 7: 42-45.

3. Elmgren L, Li X, Wilson C, Ball R, Wang J, et al. (2013) A Global Regulatory Science Agenda for Vaccines. Vaccine 31: B163-B175.

4. Immunology and Vaccine (2015) Preventable Diseases - Pink BookVaccination safety.

5. Capending MR, Tran NH, Hadinegoro SR, Ismail HIHJM, Chotpitayasunoundh T, et al. (2014) Clinical efficacy and safety of a novel tetravalent dengue vaccine in healthy children in Asia: a phase 3, randomized, observer-masked, placebo- controlled trial, Lancet 384: 1358-1365.

6. Villar L, Dayan GH, Arredondo- Garcia JL, Rivera DM, Cunha R, et al. (2015) Efficacy of a tetravalent Dengue Vaccine in Children in Latin America. N Engl J Med 372: 2.

7. Verdier F (2005) Regulatory issues in the development of new vaccines with a special emphasis on safety aspects. The Grand challenge for the future.

8. Kurokawa M, Murata R (1961) On the toxicity of the "toxoid" preparation responsible for the Kyoto catastrophe in 1948 Jpn. J Med Sci Biol 14: 249-256.

9. Fox GJ, Orlova M, Schurr E (2016) Tuberculosis in Newborns: The lessons of the Lubeck Disaster" 1929-1933. PLoS Pathog 12: e1005271.

10. Fitzpatrick $M$ (2006) The cutter incident: How America's first Polio vaccine led to a growing vaccine crisis. JRSM Med 99: 156.

11. Petricciani J, Sheets R, Griffiths E, Knezeviv I (2014) Aventitious agent in viral vaccines: Lessons learned from 4 cases. Biologicals 42: 1. 